\title{
Integrated Network Enhanced Telemetry (iNET): Impact to the Telemetry Community for the ettc2018
}

\author{
Tom Young \\ USAF Air Force Test Center, 61 N. Wolf Ave., Bldg.1633, Edwards AFB, CA., USA \\ Tommy.Young.1@us.af.mil
}

\begin{abstract}
:
The enormous increase in the complexity of modern aircraft, coupled with the Department of Defense's (DoD) spectrum reductions, has created a growing gap in DoD's ability to collect all the data needed from a flight test in real-time. Over the past 50 years, aircraft flight test performance has been monitored using a unidirectional telemetry link for the test article to the ground, sending only preplanned data to Mission Control. iNET will provide the capability to access and transmit onboard data in a packetized [Internet Protocol (IP)-based] format. This will enable flight test engineers to access aircraft data in an on-demand fashion using a duplex datalink. Without iNET, DoD ranges will be unable to meet near-term rapidly expanding test data throughput requirements, in the face of increased competition for limited radio frequency (RF) spectrum/bandwidth. Initial deployment will be at Edwards Air Force Base, California USA, and Naval Air Station (NAS) Patuxent River, Maryland USA.
\end{abstract}

This capability will provide to the test community unprecedented control from the ground over the instrumentation system on board the test article, in real-time. Based on the architecture of the system and its intended implementation, the ability to share spectrum over the radio frequency network is one of iNET's key features in support of spectral efficiency. While spectrum efficiency is a primary driver for the next generation telemetry capability, additional benefits will be realized with a two-way RF link. These benefits might initially be described as data efficiencies, however under the surface the impacts to spectrum efficiency will be extensive due to the enabling architecture. The ability to control the airborne instrumentation system from the ground, provides access not only to the data recorder but the data acquisition unit that controls data format and sample rates. This ability for dynamic real-time adjustment of information flow to the ground will change the face of flight testing in the very near future.

Key words: (bi-directional, telemetry, serial, dynamic, real-time).

\section{Background}

A continual challenge for the Aeronautical Mobil Telemetry (AMT) community is dealing with the increased data acquisition requirements imposed by aircraft information systems. With the complexity of aeronautical systems increasing, the test ranges are ever challenged to respond to this growing requirement. The pressing need to transmit massive quantities of data in real-time is the trend when supporting these next generation weapons systems. Inturn the AMT system infrastructure needs to be capable of scaling to support the needs of the these, high tech test articles. In addition to these traditional challenges, new challenges exist for AMT. The singular most significant challenge is the availability of protected test and evaluation RF spectrum allocations for AMT.

To operate in a reduced AMT spectrum environment, the end-user will need the capability to change the measurements telemetered in-flight. This capability lets the end-user start/stop the measurements associated with a specific phase of the test. To do this a two-way connection with the AMT system in the test article is needed and that does not exist in current systems. Also, once the two-way connection with the test article is established, the AMT system must be capable of accepting and responding to configuration changes. This capability also does not exist in current systems today. 
Finally, reduced spectrum means that AMT system needs to move away from command and control spectrum management. In this scheme, spectrum is allocated for each specific telemetry signal transmitted with white space between signals to avoid interference. To maximize the spectrum available, a group of test articles need to share the same spectrum with the white space recycled for use. A network enhanced AMT provides the means to modify the data telemetered to the ground inflight.
The current AMT systems architecture (see Fig. 2) does not support in-flight changes to the telemetry stream. The ability to make changes in-flight give the end-user the ability to manage what measurements are telemetered at any given time. If future AMT systems must operate with reduced spectrum then the current architecture must have the capability to make in-flight changes, the traditional architecture for AMT needs vast improvements; a shift in paradigm.

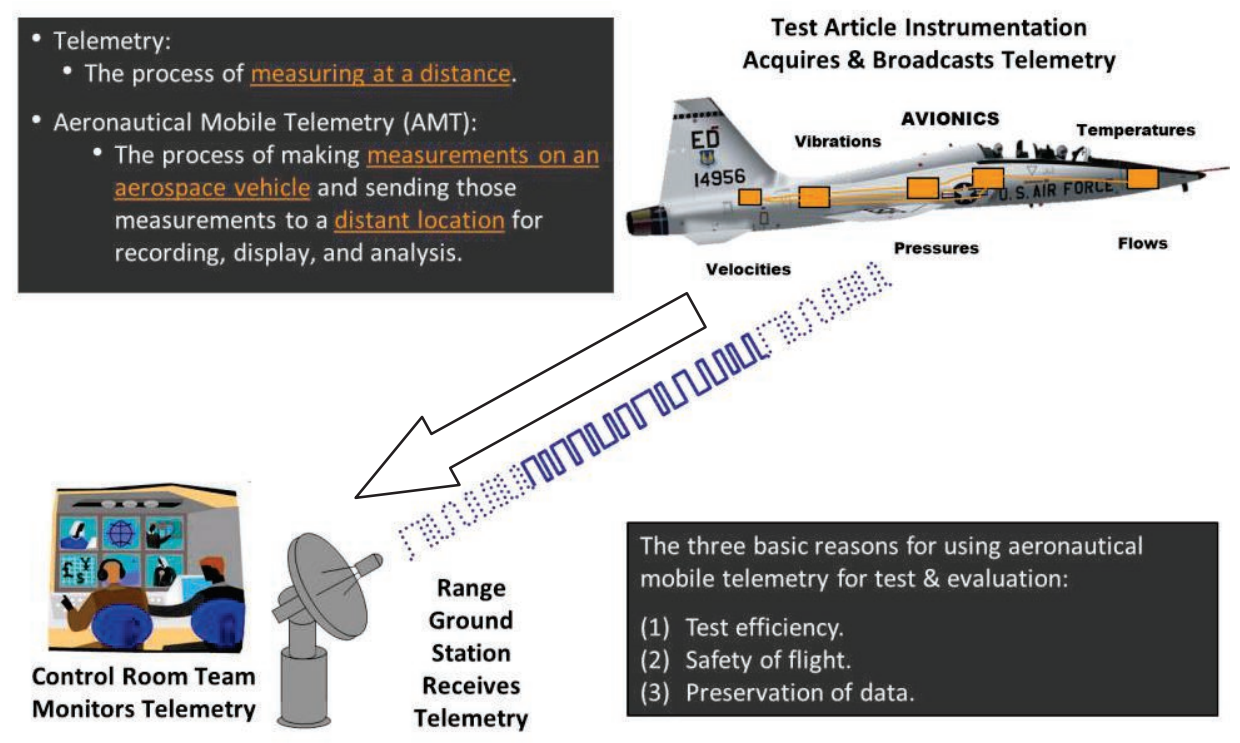

Fig.1.Current AMT Condition.

\section{Introduction}

Network telemetry is the main ingredient to ensure the shift in T\&E capability contains the needed answers to continue manned flight test at the ranges. iNET is the building block to achieve supportability of next generation weapons systems development. Without the introduction of network telemetry, many components of the T\&E infrastructure will likely be out paced by the very systems they are in place to support. Thankfully the iNET capability is nearing completion and its maturity has been tested in flight. iNET's architecture describes the standard for network telemetry within the DoD ranges, now and for the foreseeable future.

The INET capability will provide to the test community unprecedented control of the aircraft instrumentation system, from the ground in realtime. Based on the architecture of the system and its intended implementation, the ability to share spectrum over the radio frequency network is one of iNET's key features in support of spectral efficiency. Spectrum efficiency is a primary driver for the next generation telemetry capability; additional benefits will also be realized with a two-way RF link. But iNET doesn't stop there, upgrading both the System Under Test (SUT) along with the ground components complete the iNET capability. Enhancing the acquisition site with duplex communication to the aircrafts instrumentation system and providing the capacity to deconstruct TmNS network packets in a mission control room, completes the iNET system upgrades. Once the range architecture mods are in place, the full benefit of network telemetry can be realized (see Fig. 2). These modifications are the backbone of the iNET capability and provide the benefit of, spectrum efficiency, data efficiency as well as the added advantage of upgrading aging components. This, a hidden gem of the program is the requirement to provide a path to an open 


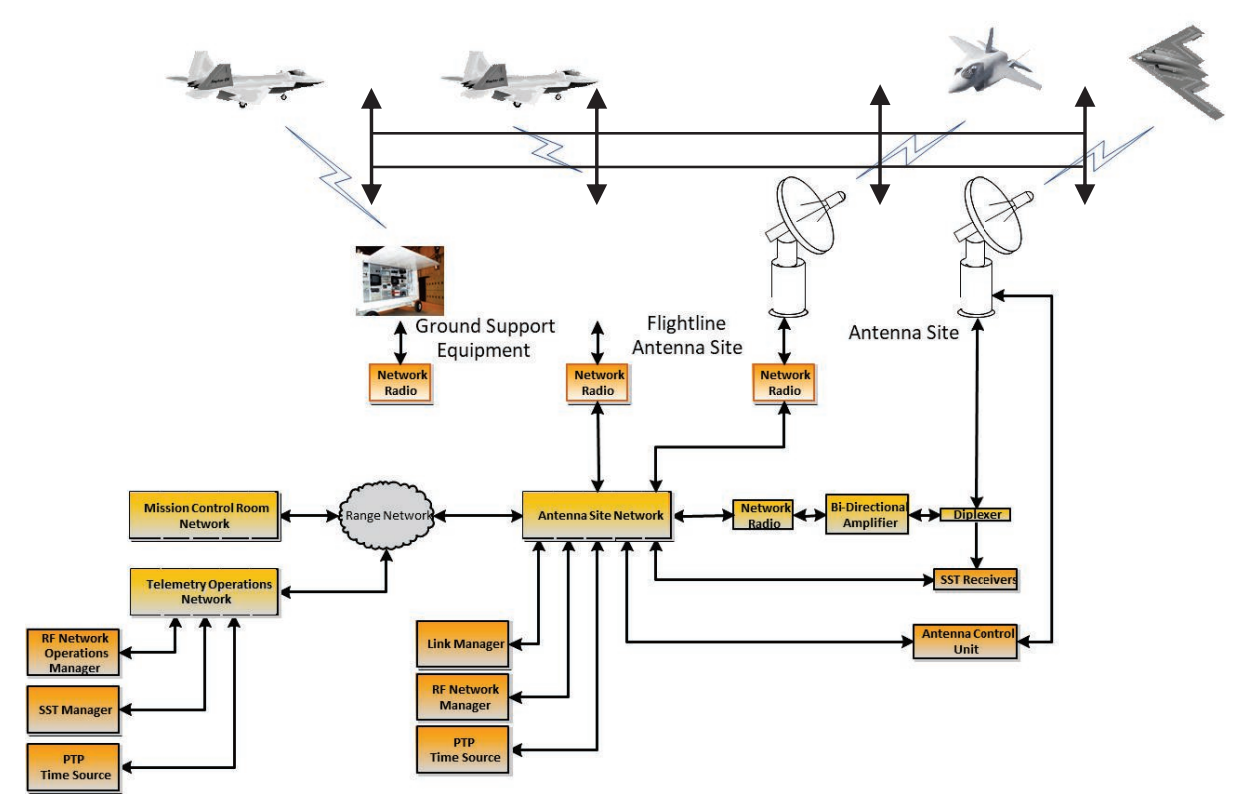

Fig.2.iNET TM Network Architecture.

architecture and move away from stove pipe proprietary solutions. By leveraging commercial standards and establishing standardized components, the goal of a low cost, interoperable system with benefits of increased spectral density and improved test data efficiency is reachable.

Impacts to spectrum efficiency will be extensive due to the enabling architecture. The ability to control the airborne instrumentation system from the ground, provides access not only to the data recorder but the data acquisition unit that controls data format and sample rates. This ability for dynamic real-time adjustment of information flow to the ground will change the face of flight testing in the very near future. Many of the technology investments being made today can enhance the DoD capability while supporting strategies to share resources without compromising test capabilities.

\section{Networked Telemetry}

The Integrated Network Enhanced Telemetry (iNET) program has developed a new approach to telemetry for the airborne environment. iNET has developed a network architecture for airborne platforms that will replace the current serial instrumentation bus with industry standard Ethernet and add a bidirectional network channel from the aircraft to the ground. While iNET will utilize Serial Streaming Telemetry (SST) links for safety of flight and other telemetry data, the addition of a networkbased communication channel will provide additional functionality that is not possible today. iNET will support command and control of the instrumentation systems while the aircraft is in flight, which will enable capabilities such as:

- Requesting data from the onboard recorder to "backfill" or replace lost or corrupt data initially transmitted over the SST link

- Changing of original SST data package or data format during flight

- Selecting the telemetry data sent down on the network link vice initial load

- Monitoring the health and status of the instrumentation system throughout the mission

- Control Airborne instrumentation system from the ground

- Requesting data from the recorder that was not originally being telemetered

iNET will use a standard network architecture that leverages the existing ground station infrastructure with minor modifications to support two-way communication. By network enabling the instrumentation systems on the aircraft as well as adding capabilities to the MCR systems supporting interactive access to testing data, the iNET program has laid the cornerstones for the modernization of AMT. This paper will build upon the enabling technologies of iNET and examine possible implementations that will enhance the duplex wireless telemetry paradigm. 


\section{Spectrum Efficiencies}

Spectrum sharing is a key aspect to iNET to reduce the spectrum requirement by introducing the concept of block allocations. The use of a Time Division Multiple Access (TDMA) architecture uses time slots versus independent frequency bands. This concept elevates the need for much of the scheduling and resource overhead associated with SST system and allows the telemetry networking tools to manage the independent links. Not only does this increase spectrum sharing but increases productivity by using the automated tools instead of manual processes. The iNET Network can be shared by up to ten test participants simultaneously, through a mix of dynamic bandwidth sharing and quality of service priority schema. Twenty Megahertz is the current agreed upon baseline network allocation, with the maximum throughput for any one link restricted to eight Megabits. The reduction in spectrum works as a function of eliminating the required guard bands between test participants, adding players increases the efficiency gained.

Another component to spectrum reduction is based on the availability of a network link; this will lead to a reduction in the SST link with a decreased amount of "critical" parameters sent down the serial link. This shift away from SST will encourage testers to reevaluate the required information being telemetered to the ground, due to having access to all the aircraft data. Throttling bandwidth based on the need or priority of the participant can also impart a reduction in spectrum required for a mission. If a priority user of the network may opt to use less bandwidth between test points knowing that bandwidth will be there when needed, allowing lesser priority testers a chance to fly and gather data.

Other artifacts of the iNET system provide command and control of the SST device allowing for power, frequency tuning and additional opportunity for enhanced modulation techniques. This in itself can reduce the spectrum footprint twenty to forty percent on the serial link by utilizing a more efficient schema. The ability to be frequency agile can additionally impact the availability of frequency resources when specific allocations become available; the transmitter can be re-tuned to the available frequency. Never before in the flight test environment has this been possible, bringing to bear usable spectrum to a test platform on demand. Automated inter-range handoff is a feature of iNET that can free-up spectrum resources and make them available to other users that much faster. Due to the manual nature of the frequency assignment process it could potentially be hours before the resource is again available for use. Not only does this autonomy provide newly released spectrum back to the resource manager quicker but also increases the opportunity for re-use.

While the above stated iNET enablers will assist the test community with the reduction of this mission critical resource, by far the most significant impact is the decrease in data being transmitted. Of course, this sounds very straightforward but until it is put into context does it sound like a revelation. In the unidirectional paradigm of current test programs, a transmitted data format is built to encompass all of the requirements of the program, from start to finish. The beauty of a two-way link is that you can organize your test program based on the spectrum needs of the specific mission, test card, test point. In other words, dynamic access to the data on the test article and only transmitting to the ground what is needed and when. That's a PARADIGM shift!

\section{Test Data Efficiencies}

Just recently, spectrum efficiency has become an extremely hot topic and has received much attention from the decision makers. Test / data efficiency has always been a recognized metric that has received considerable scrutiny. To that end an ample amount of focus has been spent increasing those metrics. Until now the previous work pales in comparison to the improvements incorporated into the Telemetry Network System (TmNS) known as "iNET". Discussed earlier as a key spectral efficiency factor, manipulation of the transmitted test data is a formidable test and data efficiency. The immediate correction of corrupt data due to link outages is the gold standard when it comes to $\mathrm{RF}$ communications. This pristine data is provided to the tester via the network data recorder in near real-time, ten to twenty milliseconds after the event. Proving to the disciplined test engineer with pristine recorder data, that the test point does not need to be reaccomplished. This technique is known as Data Backfill or SST backfill.

As important to the tester as Data Backfill, Data on Demand is the premier data efficiency feature that iNET employs. Providing the ability to request data in real-time from the onboard data recorder without interrupting recording; down to the parametric level. Access to the recorded data in real-time provides the entire data set, previously unavailable until the aircraft landed. The ability to evaluate this pristine data set in real-time can improve test efficiency by 
ensuring test points/ cards were performed to expectations; thus reducing expensive re-fly's. With the added capability of the duplex link another component that increases test efficiency is the ability to control the onboard instrumentation components. Having the ability to control instrumentation from the ground enables moving that control out of the cockpit, freeing up the pilot for more important things, flying. Additional controls allow dynamic reconfiguration of components ensuring less reflys, decreased time between missions and increased access to data more rapidly.

Due to the architecture of TmNS, managing the spectrum in chucks versus individual test frequencies significantly eases the burden of resource management. Schedulers as well as Frequency Managers will benefit from the ability to manage additional functionality with a less resources. Using the automated network tools and the TDMA building blocks increases opportunities for test and data efficiencies.

\section{Sustainability and Interoperability}

An overlooked advantage of using the open architecture, standards-based approach is cost saving, by leveraging commercial investment. Using the available network tools and standards instead of developing purpose-built tools saves time and money. The use of commercial communication standards (e.g. Ethernet, TCP/IP, UDP/IP, SNMP, RTSP, FTP, XML, PTP1588) and standard interfaces significantly decreases the acquisition, implementation and operations costs, as well as maintenance and replacement costs. These improvements alone will save the ranges considerable investment but the ability to interoperate is just as important.

The concept of test ranges being interoperable has been the charter of the Range Commanders Council (RCC). The RCC committees have done a fantastic job working together, encouraging standardization. Moving away from costly one-off proprietary solutions, building and maintaining common standards for the ranges to interoperate. Much thought was given by the initial iNET developers to develop standards for a common approach for network telemetry, defined as TMNS. These standards are captured, published and maintained by the RCC Telemetry Group and the baseline standards were released in August of 2017. These Inter-Range Instrumentation Group (IRIG) 106-17 Chapters 21-28 contain the new telemetry network standards. With the above standards the various test ranges can build, purchase, integrate and test interoperable components / systems. Interoperable capability is an enormous benefit for the test customer however cost savings is at the heart of standardization. This is the key that all members of the range community must strive to achieve when developing new capabilities.

Lastly, the vendor community involved to support interoperability. Not only are they involved in standards development but many build the components and systems to the standards. It is important to realize from a customer perspective how important it is for competition in the marketplace. Indeed, it decreases cost but also reduces the risk to the test mission support community. The reduction in risk is due to multiple vendor solutions targeting a singular problem space. Bringing the commercial community into the RCC creates cooperation and teaming for solving tough problems for the ranges. Its in the best interest for all involved to meet mission goals and at the same time be as financially responsible as possible.

\section{Implementation Considerations}

Based on the enormous change to the telemetry system that iNET provides, substantial attention needs to be focused on the Concept of Operations (ConOps). How you implement the specific network telemetry system to meet a ranges needs will certainly need consideration. A primary factor to consider is the physical placement of the control interface to the TmNS. This powerful interface will have the ability to control the instrumentation system onboard the test aircraft anytime. Concerning as this may be, determining the best location, based on the ranges needs and mission are the primary factors. Locations that have already established physical security measures in place will most likely be the locations of choice, e.g. Range Control or Mission Control Room.

An additional vital topic to be considered is where the responsibility for making control decisions will reside. Access to the network for the purpose of manipulating the transmitted data should be given to a select few. Commands requesting large amounts of data simultaneously could be detrimental to the telemetry network. Ensuring proper control of the mission data flow is essential for a successful implementation of iNET. Other considerations that need to be addressed are the modified functions of both the Resource Schedulers and the Frequency Managers. Both of these functions will be impacted by the operational network telemetry capability. Not that either of these functions will be impacted negatively, on the contrary, there is opportunity 
for increased productivity. Training on the new systems and capabilities will be an important factor for a successful transition into network telemetry. There are many new capabilities that are available with iNET and they will need specific understanding from the support organizations. System level control from a single Graphical User Interface (GUI) is powerful concept that will introduce new factors for consideration. This single system interface can also provide the capability of the current the Ground Support Equipment. Will the need for such an interface to the aircraft still exist if the GUI in the Control Facility can provide the same function? Additional opportunities to learn will present themselves as we install and learn about this new game changing capability.

Soon the test ranges will be faced with unlocking the true potential of this bi-directional telemetry architecture. Much like the advent of the internet and the cell phone, iNET has the potential to be as impactful for the telemetry community. To use an example from cellular technology; would you have guessed that the cell phones primary function is all but forgotten by todays cell phone customer. iNET provides the capacity for an application-based capability. This capability has the potential to provide unlimited test capability for the customer. Limiting the telemetry downlink because most of the decision making is taking place on the SUT, autonomously. This concept will also have an impact to instrumentation systems ultimate configuration and the acquisition sites as well. Automated tools are increasingly being used in instances where the processing horsepower was previously not available. The days of being resistant to change because its uncomfortable are over, get comfortable with these new capabilities.

\section{Conceivable Implementations}

TmNS is the platform to build upon to achieve the test capabilities required by the next generation, complex weapons systems. Without the capacity and flexibility of the TmNS, supporting telemetry requirements into the future is questionable. DoD spectrum allocations continue to decline, increasing the need for network telemetry. By continuing to apply network capability, it is possible to establish the two-way connection out to the test sensors. Enabling dynamic control out to the sensor for real-time calibration, modifying sample rate or adjusting sensitivity. Establishing real-time control out to the edges creates the environment of smart sensors.

Another conceivable implementation is on board real-time validation of models. This powerful test concept is within the art of possible, with an established duplex link. Time and cost savings can be realized as models are validated in real-time and the information is fed back to the test team. This has the implications of significantly reducing the time to test.

A distributed test capability is yet another possible implementation that is enhanced by a two-way link. The ability to send packets to any location in near real-time has great potential for cost savings. Sending test teams to various locations to conduct a test is no longer required. Sending telemetry data in realtime to a distributed test team saves time, money and possibly engineering support.

But possibly the most controversial topic to end the paper, is the ability for real-time decision automation. To potentially automate and augment the information received by the test team, increasing the speed in making engineering judgement calls. This in turn could reduce the required amount of safety information being telemetered to the ground; reducing the spectrum requirement. The possibility exists that test engineers may only have the need for $2^{\text {nd }} \& 3^{\text {rd }}$ generation processing / analysis in real-time; the rest is automated. Similar concepts are being flown today, e.g. the automated flight termination systems on unmanned vehicles.

\section{Global Ranges and Over the Horizon Coverage}

Network telemetry will also allow the realization of a "Range-less Range" or "Global Range" capability. When test articles use a packetbased infrastructure instead of the serial systems of today, testing will be conducted anywhere network connectivity is available. Options like commercial / government satellites, commercial wireless / cellular providers could be leveraged to support test missions conducted across the globe. The utilization of airborne routers and mesh or Mobile Ad-hoc NETwork (MANET) protocols could be used to extend the network where the use of satellites might not be suitable. In this case, a series of aircraft and/or surface vessels could act as mobile network routers that extend coverage over the horizon by relaying network telemetry data through each mobile node and down to the ground.

\section{Conclusion}

Clearly the current telemetry environment is ripe for technology advancement to keep pace with the next generation weapons systems development. The initial operational capability provided by iNET is the crucial building block necessary for the DoD to modernize the test 
range telemetry infrastructure. Bi-directional communication with the instrumentation system provides the improvement for the growth in capability. Controlling the data transmission to the ground is the primary driver for influencing spectrum and test efficiency goals. iNET is the capability that the DoD test community has invested in for the future of network telemetry. 